\title{
Radiation-Related Non-Neoplastic Disorder
}

National Cancer Institute

\section{Source}

National Cancer Institute. Radiation-Related Non-Neoplastic Disorder. NCI Thesaurus.

Code C53539.

A non-neoplastic disorder arising in an anatomic site exposed to radiation. 